\title{
Learning the synaptic and intrinsic membrane dynamics underlying working memory in spiking neural network models
}

\author{
Yinghao Li ${ }^{\mathbf{1}}$, Robert Kim ${ }^{\mathbf{1 , 2 , 3}}$, Terrence J. Sejnowski ${ }^{\mathbf{1 , 4}, \mathbf{5}}$ *
}

${ }^{1}$ Computational Neurobiology Laboratory, Salk Institute for Biological Studies, La Jolla, CA 92037, USA

2 Neurosciences Graduate Program, University of California San Diego, La Jolla, CA 92093, USA

3 Medical Scientist Training Program, University of California San Diego, La Jolla, CA 92093, USA

${ }^{4}$ Institute for Neural Computation, University of California San Diego, La Jolla, CA 92093, USA

${ }^{5}$ Division of Biological Sciences, University of California San Diego, La Jolla, CA 92093, USA

* Correspondence: terry@salk.edu (T.J.S.)

\section{Summary}

Recurrent neural network (RNN) model trained to perform cognitive tasks is a useful computational tool for understanding how cortical circuits execute complex computations. However, these models are often composed of units that interact with one another using continuous signals and overlook parameters intrinsic to spiking neurons. Here, we developed a method to directly train not only synaptic-related variables but also membrane-related parameters of a spiking RNN model. Training our model on a wide range of cognitive tasks resulted in diverse yet task-specific synaptic and membrane parameters. We also show that fast membrane time constants and slow synaptic decay dynamics naturally emerge from our model when it is trained on tasks associated with working memory (WM). Further dissecting the optimized parameters revealed that fast membrane properties and slow synaptic dynamics are important for encoding stimuli and WM maintenance, respectively. This approach offers a unique window into how connectivity patterns and intrinsic neuronal properties contribute to complex dynamics in neural populations. 


\section{Introduction}

2 Neurons in the cortex form recurrent connections that give rise to the complex dynamic processes

3 underlying computational functions [1-4]. Previous studies have used models based on recurrent

4 neural networks (RNNs) of continuous-rate units to characterize network dynamics behind neural

5 computations and to validate experimental findings 5 - 10 . However, these models do not explain

6 how intrinsic membrane properties could also contribute to the emerging dynamics.

$7 \quad$ Rate-based encoding of information has been reliably observed in experimental settings [8].

8 However, recent studies demonstrated that membrane potential dynamics along with spike-based

9 coding are also capable of reliably transmitting information [11 13 . In addition, the intrinsic

10 membrane properties of inhibitory neurons, including the membrane time constant and rheobase

11 (minimum current required to evoke a single action potential), were different in two higher-order

12 cortical areas [14]. These findings strongly indicate that neuronal intrinsic properties, often ignored

13 in previous computational studies employing rate-based RNNs, are crucial for better understanding

14 how distinct subtypes of neurons contribute to information processing.

15 Rate-based RNNs can be easily trained by stochastic gradient-descent to perform specified cog-

16 nitive tasks [15]. However, similar supervised learning methods cannot be used to train spiking

17 RNNs due to the non-differentiable behavior of action potentials [16]. Thus, several methods intro-

18 duced differentiable approximations of the non-differentiable spiking dynamics $17-20$. These stud-

19 ies directly applied backpropagation to tune synaptic connections for task-specific computations.

20 Other methods that do not rely on gradient computations have been also utilized to train spiking

21 networks. One such method is based on the first-order reduced and controlled error (FORCE)

22 algorithm previously developed for rate RNNs [6]. The FORCE-based methods are capable of

23 training spiking networks, but training all the parameters including recurrent connections could

24 become computationally inefficient $21-23$. Lastly, recent studies successfully converted rate-based

25 networks trained with a gradient-descent method to spiking networks for both convolutional and

26 recurrent neural networks 24,25 . Since these models are built on rate-coding networks, the result-

27 ing spiking models do not take advantage of the rich spiking dynamics. Moreover, these previous

28 models assume that all the units in a trained network are equivalent, even though experimental

29 evidence shows that neurons in biological neural networks are highly heterogeneous. Such diversity 
30 has a vital role in efficient neural coding [26].

31 Here, we present a new approach that can directly train not only recurrent synapses but also

32

\section{Results}

41 Here, we provide an overview of the method that we developed to directly train spiking recurrent 42 neural network (RNN) models (for more details see Methods). Throughout the study, we considered recurrent network models composed of leaky integrate-and-fire (LIF) units whose membrane voltage 4 dynamics were governed by:

$$
\tau_{m, i} \frac{d v_{i}}{d t}=-\left(v_{i}(t)-v_{\text {rest }_{i}}\right)+R_{i} I_{i}(t)
$$

45 where $\tau_{m, i}$ is the membrane time constant of unit $i, v_{i}(t)$ is the membrane voltage of unit $i$ at time

$46 t, v_{\text {rest }, i}$ is the resting potential of unit $i$, and $R_{i}$ is the input resistance of unit $i$. $I_{i}(t)$ represents 47 the current input to unit $i$ at time $t$, which is given by:

$$
I_{i}(t)=\sum_{j=1}^{N} s_{i j}(t)+I_{\operatorname{ext}_{i}}(t)
$$

48 where $N$ is the total number of units in the network, $s_{i j}(t)$ is the synaptic input from unit $j$ to 49 unit $i$ at time $t$, and $I_{\text {ext }, i}(t)$ is the external current source into unit $i$ at time $t$. We used a single 50 exponential synaptic filter to model the synaptic input $(s)$ :

$$
\tau_{i j} \frac{d s_{i j}}{d t}=-s_{i j}(t)+\sum_{t_{j}^{(k)}<t} w_{i j} \delta\left(t-t_{j}^{(k)}\right)
$$


51 where $\tau_{i j}$ is the decay time constant of the synaptic current from unit $j$ to unit $i, w_{i j}$ is the synaptic

52 strength from unit $j$ to unit $i, t_{j}^{(k)}$ denotes the time of the $k$-th action potential of unit $j$, and $\delta(x)$

53 is the Dirac delta function. Once the membrane voltage of the unit $i$ crosses its action potential

54 threshold $\left(\vartheta_{i}\right)$, its membrane voltage is brought back down to its reset voltage $\left(v_{\text {reset }, i}\right)$.

55 Each LIF unit is characterized by five distinct parameters: membrane time constant $\left(\tau_{m, i}\right)$, 56 resting potential $\left(v_{\text {rest }, i}\right)$, input resistance $\left(R_{i}\right)$, action potential threshold $\left(\vartheta_{i}\right)$, and reset potential $57\left(v_{\text {reset }, i}\right)$. In addition, there are two trainable synaptic parameters: synaptic strength $\left(w_{i j}\right)$ and 58 synaptic decay time constant $\left(\tau_{i j}\right)$ from unit $j$ to unit $i$.

59 In order to tune all the parameters described above to produce functional spiking RNNs capable 60 of performing cognitive tasks, we employed the commonly used gradient-descent method known as 61 backpropagation through time (BPTT; 28]) with a few important modifications. We utilized mol62 lifier gradient approximations to avoid the non-differentiability problem associated with training 63 spiking networks with backpropagation [27]. Furthermore, we optimized each of the model param64 eters (except for the synaptic connectivity weights) in a biologically plausible range (see Methods).

65 We also employed the weight parametrization method proposed by Song et al. to impose Dale's 66 principle 29] (see Methods). All the spiking RNN models trained in the study used the parameter 67 value ranges listed in Supplementary Table 1 unless otherwise noted.

68 Units with diverse parameter values emerge after training. We applied our method to train 69 spiking networks to perform the context-dependent input integration task previously employed by 70 Mante et al. 8. Briefly, Mante et al. trained rhesus monkeys to flexibly integrate sensory inputs 71 (color and motion of randomly moving dots presented on a screen). A contextual cue was given to 72 instruct the monkeys which sensory modality (color or motion) they should attend to. The monkeys 73 were required to employ flexible computations as the same modality could be either relevant or 74 irrelevant depending on the contextual cue. Several previous modeling studies have successfully 75 implemented a simplified version of the task and reproduced the neural dynamics present in the 76 experimental data with both continuous-rate RNNs and spiking RNNs converted from rate RNNs 77 25, 29, 30. With our method, we were able to directly train the first, to our knowledge, spiking 78 RNNs with heterogeneous units whose parameters were within biologically plausible limits.

79 In order to train spiking RNNs to perform the input integration task, we employed a task 
80 paradigm similar to the one used by previous computational studies [8, 25, 29, 30]. A recurrently

81 connected network received two streams of noisy input signals along with a constant-valued signal

82 that encoded the contextual cue (Fig. 1A). The input signals were sampled from a standard Gaus-

83 sian distribution (i.e., with zero mean and unit variance) and then shifted by a positive or negative

84 "offset" value to simulate the evidence presented in the input modalities. The network was trained

85 to produce an output signal approaching either +1 or -1 depending on the cue and the evidence

86 present in the input signal: if the cued input had a positive mean, the output signal approached

$87+1$, and vice versa ( $\overline{\text { Fig. 1B }}$ top). The input signal, $150 \mathrm{~ms}$ in duration, was given after a fixation

88 period $(300 \mathrm{~ms})$, and the network was trained to produce an output signal immediately after the

89 offset of the input signal.

90 We trained 20 spiking RNNs to perform the context-based input integration task. All the train91 able parameters were initialized with random numbers drawn from a standard Gaussian distribution 92 and re-scaled to the biologically plausible ranges (see Methods and Supplementary Table 1). Each 93 network was trained until the training termination criteria were satisfied (see Methods). On av94 erage, $508.21 \pm 45.96$ training trials were needed for a network to meet the training termination conditions. After training, a wide distribution of the parameters emerged for both excitatory and inhibitory populations (Fig. 1C, top).

$97 \quad$ Consistent with the previous experimental recordings from cortical neurons, the inhibitory units

98 in our trained RNNs fired at a higher rate compared to the excitatory units [31]. The higher average

99 firing rates of the inhibitory units were largely due to the intrinsic properties that resulted from

100 training. Compared to the excitatory population, the inhibitory units in the trained RNNs had

101 significantly larger input resistance, smaller membrane time constants, and more depolarized resting

102 potential (Fig. 1C; $P<0.0001$, two-sided Wilcoxon rank-sum test). The action potential thresholds

103 and the reset potentials were significantly more depolarized for the inhibitory group. Furthermore,

104 the time constants of the inhibitory synaptic current variable were significantly larger than the 105 excitatory synaptic decay time constants (Fig. 1C).

106 Working memory requires distinct parameter distributions. The context-dependent input

107 integration task considered in the previous section did not require complex cognitive skills such as 108 working memory (WM) computations. In order to explore what parameter values are essential for 
109 WM tasks, we modified the paradigm to incorporate a WM component by adding a delay period

110 after the delivery of the input signals. The RNN model was trained to integrate the noisy input

111 signals, sustain the integrated information throughout the $300 \mathrm{~ms}$ delay period, and produce an

112 output signal (Fig. 1B bottom). We again trained 20 models for the modified integration task with

113 the same training termination criteria (see Methods). This task required more training trials (on

114 average $1618.10 \pm 345.54$ ), but all the models were successfully trained within 2000 training trials.

115 Overall, the distributions of the trained parameters were similar to those observed from the

116 RNNs trained on the non-WM version of the task (Fig. 1D). The parameters that were significantly

117 different between the two RNN models were the membrane time constant and the synaptic decay

118 time constant. The inhibitory units from the WM model displayed much faster membrane dynamics

119 and slower synaptic decay compared to the inhibitory population of the non-WM model $(P<$

120 0.0001, two-sided Wilcoxon rank-sum test).

121 To ensure that the patterns of the trained parameters and the distinct distributions of the two

122 parameters $\left(\tau_{m}\right.$ and $\left.\tau\right)$ observed from the delayed integration model were indeed associated with

123 WM computations, we trained RNNs on two additional WM-related tasks: delayed matched-to-

124 sample (DMS) and delayed discrimination (DIS) tasks. For each task, we again trained 20 RNNs.

125 Both task paradigms included two sequential stimuli separated by a brief delay period. For the

126 DMS task, the two input stimuli were either +1 or -1 ; if the two sequential had the same sign

127 (i.e., $+1 /+1$ or $-1 /-1$ ), the network was trained to have an output signal approaching +1 ,

128 while if the two stimuli had different signs (i.e., $+1 /-1$ or $-1 /+1$ ), the output signal approached

$129-1$ (Fig. 2A; see Methods). The two input stimuli for the DIS task were sinusoidal waves with

130 different frequencies, modeled after the task used by Romo et al. [32] where monkeys were trained

131 to discriminate two vibratory stimuli. If the first stimulus had a higher (lower) frequency, our RNN

132 model was trained to produce a positive (negative) output signal (Fig. 2B; see Methods).

133 It took longer to train our model on these two tasks compared to the delayed integration task

134 (7103.95 \pm 3738.65 trials for the DMS task and $6985.47 \pm 2112.34$ trials for the DIS task). The

135 distributions of the tuned parameters from the two WM tasks were similar to the distributions

136 obtained from the delayed integration task (Fig. 2 $\mathrm{C}$ and D). More importantly, we again observed

137 significantly faster membrane voltage dynamics and slower synaptic decay from the inhibitory 
138 units in the DMS and DIS models compared to the inhibitory units from the non-WM task. These

139 findings strongly suggest that the two parameters $\left(\tau_{m}\right.$ and $\left.\tau\right)$ of the inhibitory group contribute to

140 important dynamics associated with WM.

141 Shared intrinsic properties across different working memory tasks. Prefrontal cortex and

142 other higher-order cortical areas have been shown to integrate information in a flexible manner and

143 switch between tasks seamlessly [8]. Along this line of thought, we hypothesized that the intrinsic

144 properties optimized for one WM task should be generalizable to other tasks that also require

145 WM. In order to test this hypothesis, we re-trained all the RNNs that were trained in the previous

146 sections to perform the DMS task without tuning the intrinsic parameters. For example, given a

147 network trained on the non-WM integration task, we froze its intrinsic $\left(R, \tau_{m}, v_{\text {rest }}, v_{\text {reset }} \vartheta\right)$ along

148 with the synaptic decay time constant $(\tau)$ and optimized the recurrent connections $(W)$ only using

149 BPTT (see Methods). Therefore, each of the 20 RNNs trained for each of the four tasks (non-WM

150 integration, delayed integration, DMS, and DIS tasks) was re-trained to perform the DMS task.

151 As expected, the average number of trials required to successfully retrain the RNNs previously

152 trained for the DMS task was low at $4408.95 \pm 3596.27$ (Fig. 3A). The number of trials required

153 to re-train the RNNs from the DIS task was also low at $4180.30 \pm 2692.81$. The RNNs trained

154 for the delayed integration task took longer to re-train at $5391.85 \pm 2197.99$. The non-WM RNNs

155 required the most number of training trials to perform the DMS task (9647.55 \pm 2933.17$)$. These

156 findings indicate that the intrinsic properties from one WM model are transferable to other WM

157 models.

158 Based on these previous results, the membrane time constant $\left(\tau_{m}\right)$ and the synaptic decay $(\tau)$

159 variables appeared to be the two most important parameters for the transferability of WM. To test

160 this, we repeated the re-training procedure with both $\tau_{m}$ and $\tau$ either fixed ("frozen") or optimized

161 ("tuned") for the non-WM RNNs (see Methods). For the "frozen" condition (i.e., $\tau_{m}$ and $\tau$ frozen

162 while the other parameters optimized), the number of trials required to re-train the non-WM RNNs

163 to perform the DMS task was high and not significantly different from the number of trials it took

164 with the intrinsic parameters fixed $($ Fig. $3 B$ B $)$. On the other hand, re-tuning only $\tau_{m}$ and $\tau$ with the

165 other parameters fixed (i.e., "tuned" condition) resulted in a significant reduction in training time

166 (Fig. 3B), suggesting that these two parameters are indeed critical for performing WM. Optimizing 
167 both $\tau_{m}$ and $\tau$ resulted in a significant decrease in $\tau_{m}$ for both excitatory and inhibitory populations

168 (Fig. 3C). The synaptic decay values decreased for the excitatory units after re-tuning (Fig. 3D

169 left). For the inhibitory population, $\tau$ was significantly increased (Fig. 3D right).

170 Membrane and synaptic decay time constants critical for WM maintenance. Pyramidal

171 excitatory neurons and parvalbumin (PV) interneurons make up the majority of the neuronal cell

172 population in the cortex, and they have been shown to specialize in fast and reliable encoding of

173 information with high temporal precision 33. To further investigate if the fast membrane and

174 slow synaptic dynamics of the units from our WM RNNs are aligned with previous experimental

175 findings and to probe how they contribute to WM maintenance, we manipulated $\tau_{m}$ and $\tau$ during

176 different epochs of the DMS task paradigm.

177 For each of the RNNs trained from the DMS task, we first divided the population into two

178 subgroups based on their $\tau_{m}$ values (see Methods). The short $\tau_{m}$ group contained units whose $\tau_{m}$

179 was smaller than the lower quartile value, while the long $\tau_{m}$ group contained units whose $\tau_{m}$ was

180 greater than the upper quartile. During each of the four epochs (fixation, first stimulus, delay, and

181 second stimulus), we then inhibited the two $\tau_{m}$ subgroups separately by hyperpolarizing them and

182 assessed the task performance (see Methods). As shown in Fig. 4 inhibiting the short $\tau_{m}$ subgroup

183 during the two stimulus windows significantly impaired task performance (Fig. 4 $B$ and D), while

184 disrupting the long $\tau_{m}$ group did not result in significant changes in task performance in all four

185 task epochs.

186 We repeated the above analysis with two subgroups derived from a quartile split of the synaptic

187 decay time constant ( $\tau$; see Methods). Suppressing the synaptic connections in the long $\tau$ subgroup

188 during the first stimulus window and the delay period significantly impaired task performance

189 (Fig. 4B and C). Inhibiting the short $\tau$ group at any of the four epochs did not affect the task

190 performance.

191 Therefore, the units with the fast membrane voltage dynamics $\left(\tau_{m}\right)$ were important for encoding

192 of stimuli, while the slow synaptic dynamics $(\tau)$ were critical for maintaining the first stimulus

193 information throughout the period spanning from the first stimulus window to the end of the delay

194 window. 


\section{Discussion}

196 In this study, we presented a new method for directly training spiking RNNs with a gradient-based

197 supervised training algorithm. Our approach allows optimizing not only the synaptic variables but

198 also parameters intrinsic to spiking dynamics. By optimizing a wide range of parameters, we first

199 demonstrated that units with diverse features emerged when the model was trained on a cognitive

200 task (Figs. 1 and 2). We also showed that fast membrane dynamics combined with a slow synaptic

201 property are critical for performing WM tasks (Figs. 3 and 4). Diversity is a basic biological

202 principle that emerged here as a basic computational principle in spiking neural models.

203 Previous modeling studies have trained RNNs to perform cognitive tasks [8, 34, 35]. Although

204 some of these studies were able to train spiking RNN models, the intrinsic parameters of spiking

205 neurons were not included as trainable variables. By using the mollifier approximation [27], we

206 developed a comprehensive framework that can tune both connectivity and spiking parameters

207 using a gradient-descent method. Training spiking RNNs on multiple tasks using our method

208 revealed functional specialization of excitatory and inhibitory neurons. More importantly, our

209 approach allowed us to identify fast membrane voltage dynamics as an essential property required

210 to encode incoming stimuli robustly for WM tasks.

211 Previous computational studies employing RNNs assumed that all the units in a network shared

212 the same intrinsic parameters and optimized only synaptic connectivity patterns during training.

213 Recent studies developed models that give rise to units with heterogeneous intrinsic properties.

214 For example, a new activation function that is tunable for each neuron in a network was recently

215 proposed [36]. In addition, we recently trained synaptic decay time constants in a rate RNN model

216 25]. Although these methods produce heterogeneous units, they do not incorporate parameters

217 inherent to spiking mechanisms. Our method not only allows direct training of synaptic weights

218 of spiking RNNs that abide by Dale's principle, but also enables training of synaptic and intrinsic

219 membrane parameters for each neuron.

220 Although our method was successful at training spiking RNNs with biological constraints, the 221 gradient-based method employed in the present study is not biologically plausible. In cortical neural 222 networks, local learning rules, such as spike-timing-dependent plasticity (STDP), were observed,

223 but the gradient-descent algorithm used in our method is neither local to synapses nor local in time 
224 16. However, this non-locality allowed our method to train intrinsic membrane and connectivity

225 parameters, creating biologically plausible neural architectures that solve specified problems. The

226 learning algorithm for spiking neurons makes it possible to uncover neural dynamics hidden in

227 experimental data $8,29,37$, thus emphasizing that a biologically realistic model can be constructed

228 by non-biological means.

229 Another limitation of our framework arises from our spiking neuron model. Although we were

230 able to train models with heterogeneous neurons the leaky integrate-and-fire model used in the

231 present study can only capture the dynamics of fast-firing neurons due to the lack of adaptation

232 [38. In particular, several other types of neurons, such as regular-firing and bursting neurons,

233 are also common in cortical networks [39]. Applying our method to spiking neuron models with

234 adaptation currents, such as those in Hodgkin-Huxley models model [40] and adaptive exponential

235 integrate-and-fire model [41], will be an interesting next step to further investigate the role of

236 neurons from various firing classes in information processing.

237 In summary, we provide a novel approach for directly training both connectivity and membrane

238 parameters in spiking RNNs. Training connectivity and intrinsic membrane parameters revealed

239 distinct populations only identifiable by their parameter values, thus enabling investigation of the

240 roles played by specific populations in the computation processes. This lays the foundation for

241 uncovering how neural circuits process information with discrete spikes and building more power-

242 efficient spiking networks. 
A

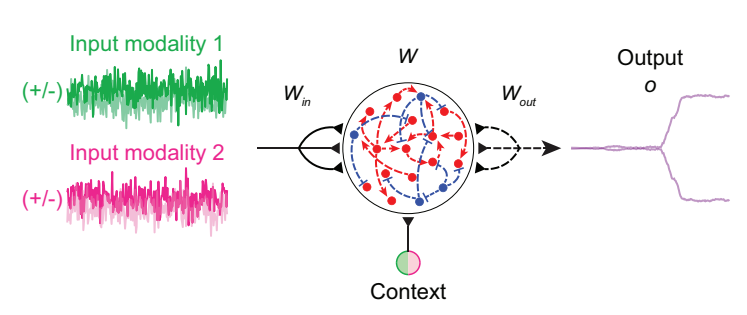

B
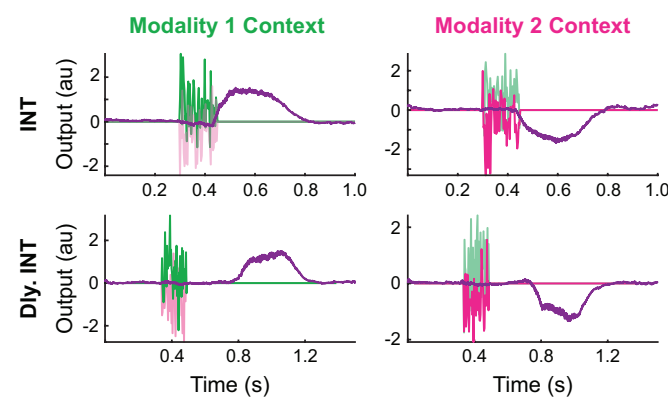
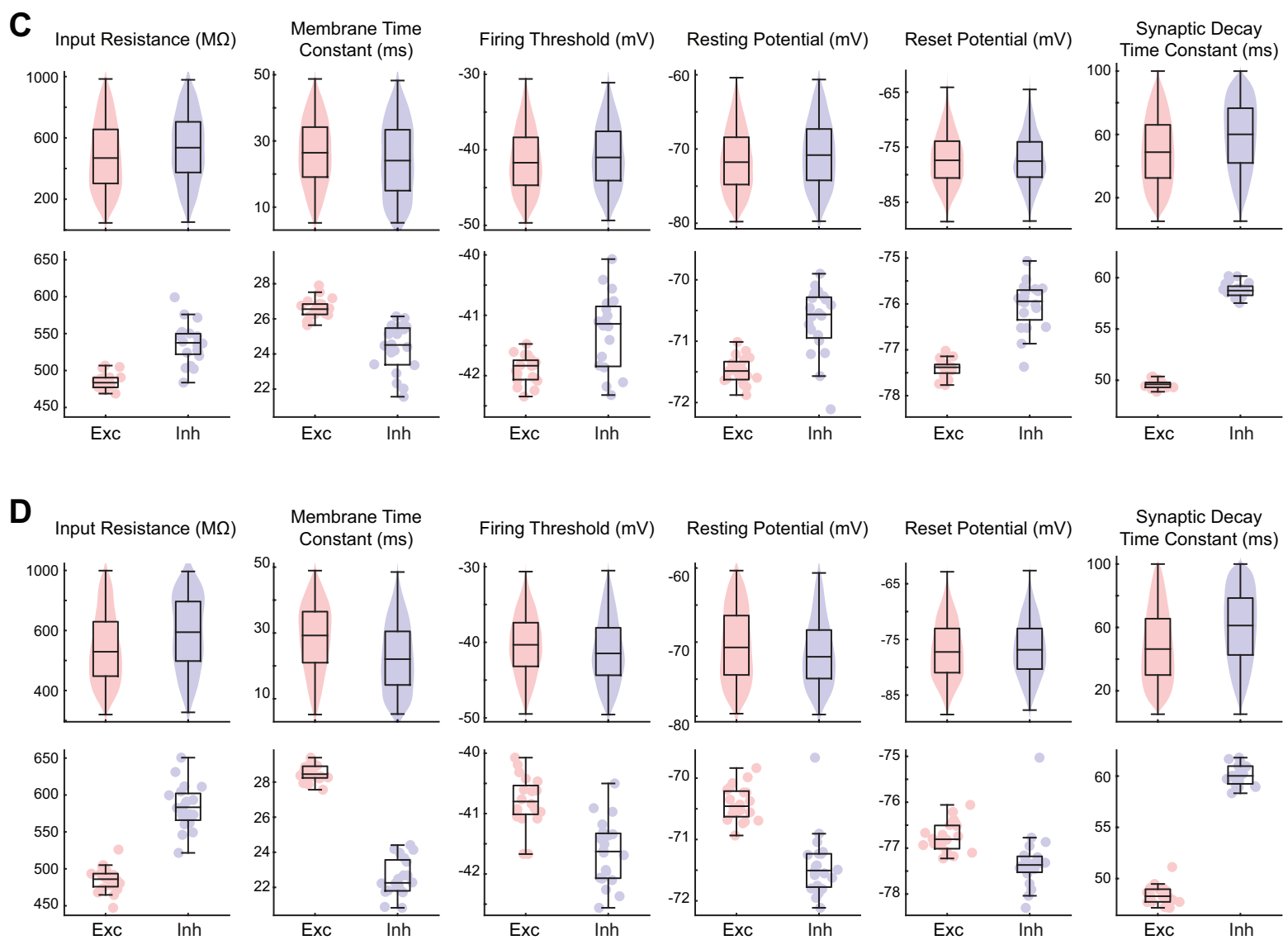

Fig. 1 | Biologically realistic spiking network performing a context-dependent input integration task. (A) Schematic diagram of the RNN model trained for the context-dependent integration task. Two streams of noisy input signals (green and magenta lines) along with a context signal were delivered to the LIF RNN. The network was trained to integrate and determine if the mean of the cued input signal (i.e., cued offset value) was positive ("+" choice) or negative ("-" choice) without or with a delay period at the end of the noisy input signals. (B) Example input and output signals from example RNNs trained to perform the task without (top row; INT) or with a delay period (bottom row; Dly. INT). (C) Distributions of the optimized parameters for the excitatory (red) and inhibitory (blue) units across all 20 models trained for the INT task. Top, distributions pooled from all the units from 20 models. Bottom, each dot represents the average value from one network. (D) Distributions of the optimized parameters for the excitatory (red) and inhibitory (blue) units across all 20 models trained for the Dly. INT task. Top, distributions pooled from all the units from 20 models. Bottom, each dot represents the average value from one network. 
A
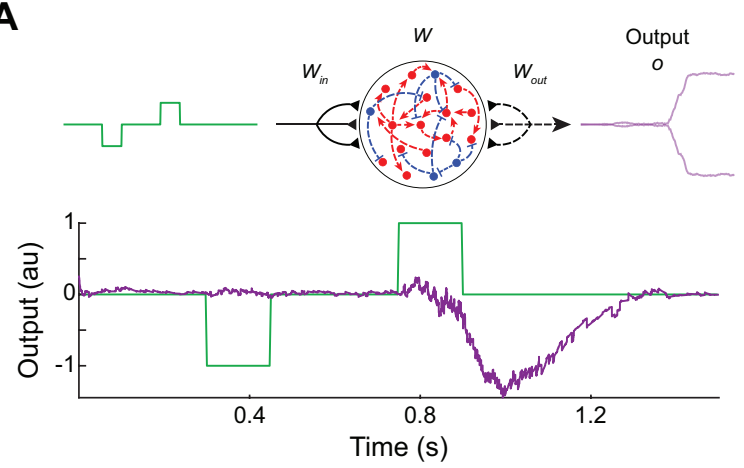

C
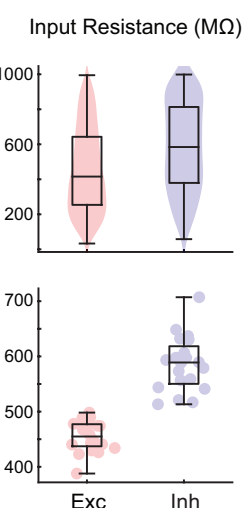

D
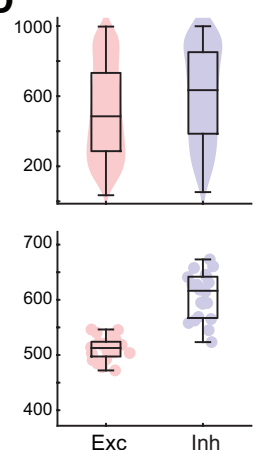

Membrane Time
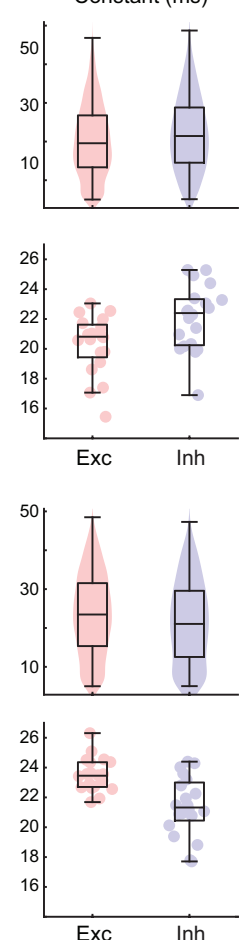

B
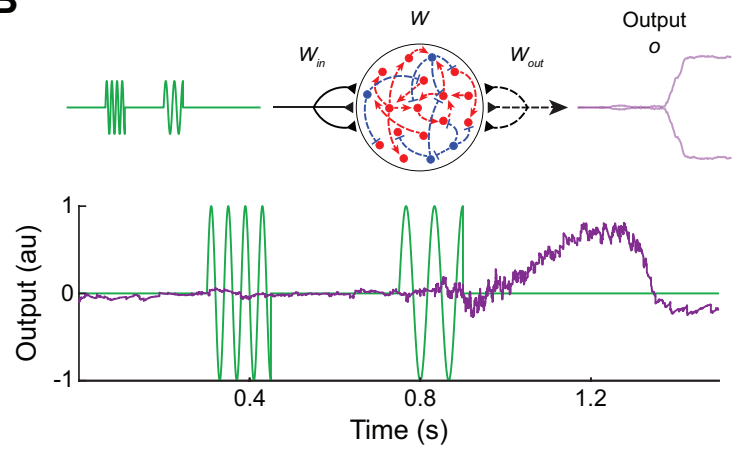

Firing Threshold (mV)

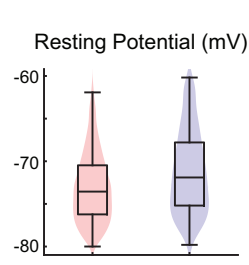

Reset Potential $(\mathrm{mV})$
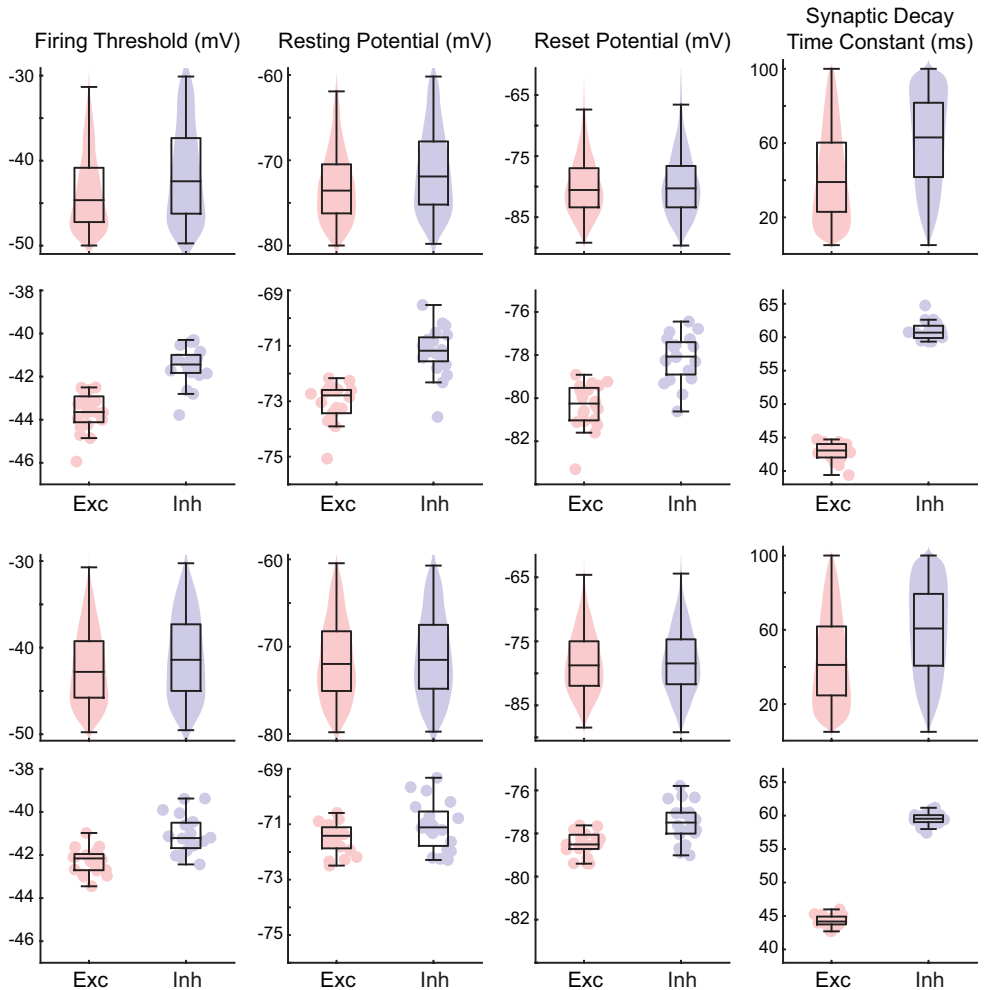

Fig. 2 | RNNs trained for two additional WM tasks. (A) Schematic illustrating the task paradigm for the delayed match-to-sample (DMS) task (top) and input and output signals from an example trained RNN (bottom). (B) Schematic illustrating the task paradigm for the delayed discrimination (DIS) task (top) and input and output signals from an example trained RNN (bottom). (C) Distributions of the optimized parameters for the excitatory (red) and inhibitory (blue) units across all 20 models trained for the DMS task. Top, distributions pooled from all the units from 20 models. Bottom, each dot represents the average value from one network. (D) Distributions of the optimized parameters for the excitatory (red) and inhibitory (blue) units across all 20 models trained for the DIS task. Top, distributions pooled from all the units from 20 models. Bottom, each dot represents the average value from one network. 


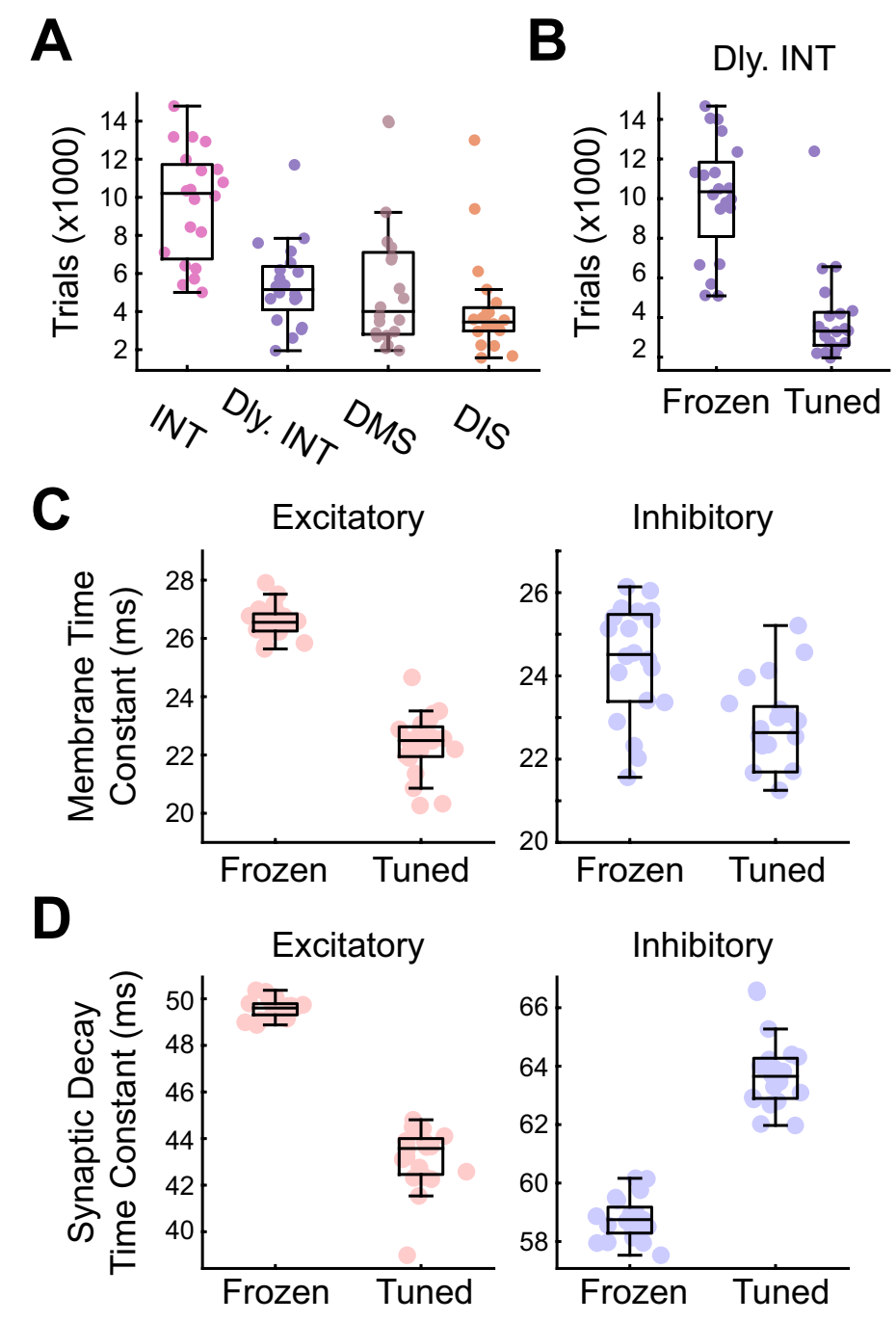

Fig. 3 | Retraining RNN models to perform the DMS task. (A) Number of training trials required to retrain the models previously trained for the INT, Dly. INT, DMS, or DIS tasks to perform the DMS task. (B) Number of training trials required to retrain the Dly. INT RNNs to perform the DMS task with the membrane time constant $\left(\tau_{m}\right)$ and synaptic decay time constant $(\tau)$ frozen or tuned. (C) Distribution of the membrane time constant values for the excitatory (red) and inhibitory (blue) units for the two conditions (frozen and tuned). Each dot represents the average value from one network. (D) Distribution of the synaptic decay time constant values for the excitatory (red) and inhibitory (blue) units for the two conditions (frozen and tuned). Each dot represents the average value from one network. 
A

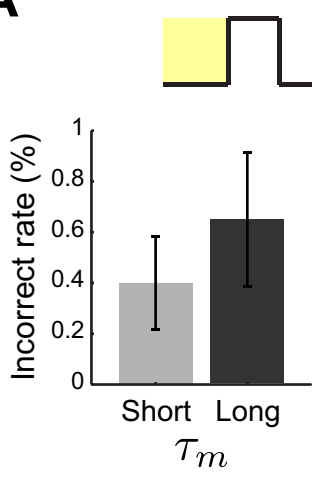

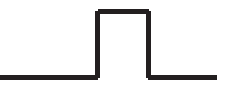

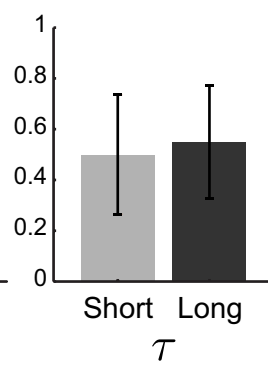

C

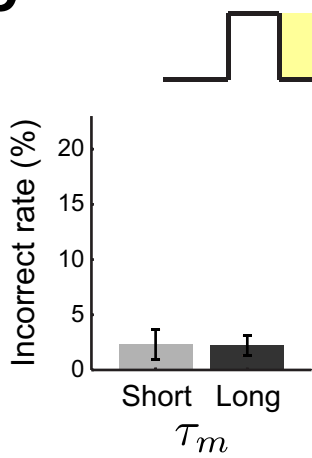

B

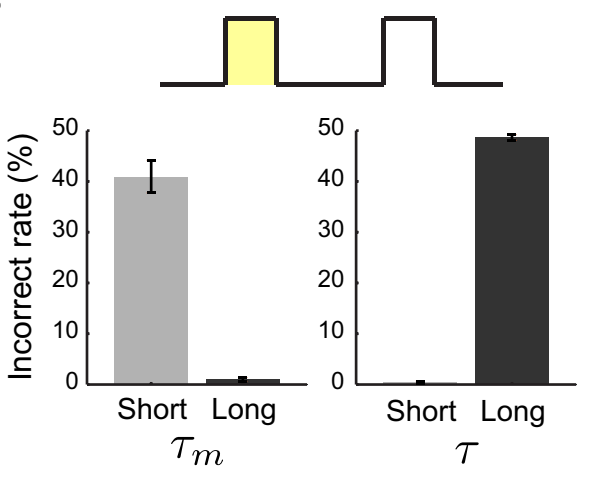

D

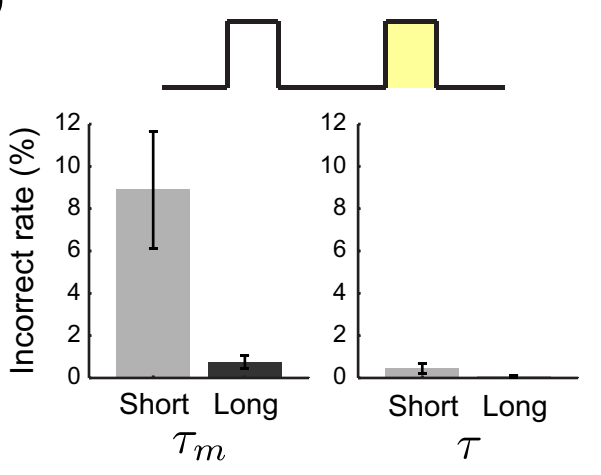

Fig. 4 | Membrane and synaptic time constants important for encoding stimuli and WM maintenance. (A-D) DMS task performance when short $\tau_{m}$, long $\tau_{m}$, short $\tau$, or long $\tau$ units were inhibited during the fixation (A), first stimulus window (B), delay period (C), or second stimulus window (D). 


\section{References}

244 1. Goldman-Rakic, P. S. Cellular basis of working memory. Neuron 14, 477-485 (1995).

245 2. Chen, L. \& Aihara, K. Chaotic simulated annealing by a neural network model with transient $246 \quad$ chaos. Neural networks 8, 915-930 (1995).

247 3. Douglas, R. J. \& Martin, K. A. Recurrent neuronal circuits in the neocortex. Current biology $248 \quad$ 17, R496-R500 (2007).

249 4. Wang, X.-J. Decision making in recurrent neuronal circuits. Neuron 60, 215-234 (2008).

250 5. Sompolinsky, H., Crisanti, A. \& Sommers, H.-J. Chaos in random neural networks. Physical $251 \quad$ review letters $\mathbf{6 1}, 259$ (1988).

252 6. Sussillo, D. \& Abbott, L. F. Generating coherent patterns of activity from chaotic neural $253 \quad$ networks. Neuron 63, 544-557 (2009).

7. Rajan, K., Abbott, L. \& Sompolinsky, H. Stimulus-dependent suppression of chaos in recurrent neural networks. Physical review e 82, 011903 (2010).

8. Mante, V., Sussillo, D., Shenoy, K. V. \& Newsome, W. T. Context-dependent computation by recurrent dynamics in prefrontal cortex. Nature 503, 78 (2013).

9. Mastrogiuseppe, F. \& Ostojic, S. Linking connectivity, dynamics, and computations in lowrank recurrent neural networks. Neuron 99, 609-623 (2018).

10. Rajan, K., Harvey, C. D. \& Tank, D. W. Recurrent network models of sequence generation and memory. Neuron 90, 128-142 (2016).

11. VanRullen, R., Guyonneau, R. \& Thorpe, S. J. Spike times make sense. Trends in neurosciences 28, 1-4 (2005).

264 12. Sippy, T., Lapray, D., Crochet, S. \& Petersen, C. C. Cell-type-specific sensorimotor processing in striatal projection neurons during goal-directed behavior. Neuron 88, 298 -305 (2015).

266 13. Pala, A. \& Petersen, C. C. State-dependent cell-type-specific membrane potential dynamics and unitary synaptic inputs in awake mice. Elife 7 (eds Dan, Y. \& Marder, E.) e35869 (2018). 
14. Medalla, M., Gilman, J. P., Wang, J.-Y. \& Luebke, J. I. Strength and diversity of inhibitory signaling differentiates primate anterior cingulate from lateral prefrontal cortex. Journal of neuroscience 37, 4717-4734 (2017).

15. Rumelhart, D. E., Hinton, G. E., Williams, R. J., et al. Learning representations by backpropagating errors. Cognitive modeling 5, 1 (1988).

16. Tavanaei, A., Ghodrati, M., Kheradpisheh, S. R., Masquelier, T. \& Maida, A. Deep learning in spiking neural networks. Neural networks (2018).

17. Lee, J. H., Delbruck, T. \& Pfeiffer, M. Training deep spiking neural networks using backpropagation. Frontiers in neuroscience 10, 508 (2016).

18. Huh, D. \& Sejnowski, T. J. Gradient descent for spiking neural networks in Advances in neural information processing systems (2018), 1433-1443.

19. Zhang, W. \& Li, P. Spike-train level backpropagation for training deep recurrent spiking neural networks in Advances in neural information processing systems (2019), 7800-7811.

20. Neftci, E. O., Mostafa, H. \& Zenke, F. Surrogate gradient learning in spiking neural networks. Corr abs/1901.09948. arXiv: 1901.09948 (2019).

21. Kim, C. M. \& Chow, C. C. Learning recurrent dynamics in spiking networks. Elife 7 (2018).

22. Thalmeier, D., Uhlmann, M., Kappen, H. J. \& Memmesheimer, R.-M. Learning universal computations with spikes. Plos computational biology 12 (ed Bethge, M.) e1004895 (2016).

23. Nicola, W. \& Clopath, C. Supervised learning in spiking neural networks with force training. Nature communications 8, 2208 (2017).

24. Sengupta, A., Ye, Y., Wang, R., Liu, C. \& Roy, K. Going deeper in spiking neural networks: vgg and residual architectures. Frontiers in neuroscience 13 (2019).

25. Kim, R., Li, Y. \& Sejnowski, T. J. Simple framework for constructing functional spiking recurrent neural networks. Proceedings of the national academy of sciences 116, 22811-22820 (2019).

26. Chelaru, M. I. \& Dragoi, V. Efficient coding in heterogeneous neuronal populations. Proceedings of the national academy of sciences 105, 16344-16349 (2008). 
295 27. Ermoliev, Y. M., Norkin, V. I. \& Wets, R. J. The minimization of semicontinuous functions: mollifier subgradients. Siam journal on control and optimization 33, 149-167 (1995).

297 28. Werbos, P. J. et al. Backpropagation through time: what it does and how to do it. Proceedings 298 of the ieee $\mathbf{7 8}, 1550-1560$ (1990).

299 29. Song, H. F., Yang, G. R. \& Wang, X.-J. Training excitatory-inhibitory recurrent neural networks for cognitive tasks: a simple and flexible framework. Plos computational biology 12, e1004792 (2016).

30. Miconi, T. Biologically plausible learning in recurrent neural networks reproduces neural dynamics observed during cognitive tasks. Elife 6, e20899 (2017).

31. Peyrache, A. et al. Spatiotemporal dynamics of neocortical excitation and inhibition during human sleep. Proceedings of the national academy of sciences 109, 1731-1736 (2012). memory in the prefrontal cortex. Nature 399, 470-473 (1999).

33. Tremblay, R., Lee, S. \& Rudy, B. Gabaergic interneurons in the neocortex: from cellular properties to circuits. Neuron 91, 260-292 (2016).

34. Song, H. F., Yang, G. R. \& Wang, X.-J. Training excitatory-inhibitory recurrent neural networks for cognitive tasks: a simple and flexible framework. Plos computational biology 12 (ed Sporns, O.) e1004792 (2016).

35. Miconi, T. Biologically plausible learning in recurrent neural networks reproduces neural dynamics observed during cognitive tasks. Elife 6 (ed Frank, M. J.) e20899 (2017). arxiv:1710.05941 (2017).

37. Remington, E. D., Narain, D., Hosseini, E. A. \& Jazayeri, M. Flexible sensorimotor computations through rapid reconfiguration of cortical dynamics. Neuron 98, 1005-1019 (2018). Press, 2014). 


\section{Acknowledgements}

334 We are grateful to Jorge Aldana for assistance with computing resources. This work was funded by 335 the DARPA (W911NF1820259 to TJS) and National Institute of Mental Health (F30MH115605336 01A1 to R.K.). We also gratefully acknowledge the support of NVIDIA Corporation with the 337 donation of the Quadro P6000 GPU used for this research. The funders had no role in study 338 design, data collection and analysis, decision to publish, or preparation of the manuscript.

\section{Author contributions}

340 Y.L., R.K., and T.J.S. designed the study and wrote the manuscript. Y.L. performed the analyses 341 and simulations.

\section{Declaration of interests}

343 The authors declare no competing interests. 


\section{Methods}

345 Spiking network structure and discretization. Our spiking RNN model consisted of $N$ integrate-

346 and-fire (LIF) units is governed by

$$
\tau_{m, i} \frac{d v_{i}}{d t}=-\left(v_{i}(t)-v_{\text {rest }_{i}}\right)+R_{i} I_{i}(t)+\xi
$$

347 where $\tau_{m, i}$ is the membrane time constant of unit $i, v_{i}(t)$ is the membrane voltage of unit $i$ at

348 time $t, v_{\text {rest }, i}$ is the resting potential of unit $i$, and $R_{i}$ is the input resistance of unit $i$, and $\xi$ is the

349 membrane voltage spontaneous fluctuation. $I_{i}(t)$ represents the current input to unit $i$ at time $t$,

350 which is given by:

$$
I_{i}(t)=\sum_{j=1}^{N} s_{i j}(t)+I_{\operatorname{ext}_{i}}(t)
$$

351 where $N$ is the total number of units in the network, $s_{i j}(t)$ is the filtered spike train of unit $j$ to

352 unit $i$ at time $t$, and $I_{\text {ext }_{i}}(t)$ is the external current source into unit $i$ at time $t$. For this study, $353 N=400$ for all tasks and networks trained.

The external current $\boldsymbol{I}_{\text {ext }}(t)$ encodes the task-specific input at time $t$ :

$$
\boldsymbol{I}_{\text {ext }}(t)=W_{\text {in }} \boldsymbol{u}(t)
$$

355 where the time-varying stimulus signals $\boldsymbol{u}(t) \in \mathbb{R}^{N_{\text {in }} \times 1}$ are fed into the network via $W_{\text {in }} \in \mathbb{R}^{N \times N_{\text {in }}}$,

356 which can be viewed as presynaptic connections to the network that convert analog input into firing 357 rates. $N_{\text {in }}$ corresponds to the number of channels in the input signal.

358 We used a single exponential synaptic filter:

$$
\tau_{i j} \frac{d s_{i j}}{d t}=-s_{i j}(t)+\sum_{t_{j}^{(k)}<t} w_{i j} \delta\left(t-t_{j}^{(k)}\right)
$$

359 where $\tau_{i j}$ is the synaptic decay time constant from unit $j$ to unit $i, w_{i j}$ is the synaptic strength

360 from unit $j$ to unit $i, t_{j}^{(k)}$ denotes the time of the $k$-th action potential of unit $j$, and $\delta(x)$ is the

361 Dirac delta function. Once the membrane voltage of the unit $i$ crosses its action potential threshold

$362\left(\vartheta_{i}\right)$, its membrane voltage is brought back down to its reset voltage $\left(v_{\text {reset }, i}\right)$. 


$$
o(t)=W_{\text {out }} \boldsymbol{r}(t)
$$

364 where $W_{\text {out }} \in \mathbb{R}^{1 \times N}$ are the readout weights, and $\boldsymbol{r}(t) \in \mathbb{R}^{N \times 1}$, which can be interpreted as the 365 firing rate of units, are given by

$$
\tau_{r, i} \frac{d r_{i}}{d t}=-r_{i}(t)+\sum_{t_{i}^{(k)}<t} \delta\left(t-t_{i}^{(k)}\right)
$$

366 where $\tau_{r, i}$ is the synaptic decay time constant of firing rate estimate for unit $i$.

367 We converted the continuous-time differential equations to discrete-time iterative equations 368 and used numerical integration (Euler's method) to solve the equations. The membrane voltage $369 \boldsymbol{v} \in \mathbb{R}^{1 \times N}$ at step $n+1$ is given by

$$
\boldsymbol{v}^{(n+1)}=\tilde{\boldsymbol{v}}^{(n)}+\frac{\Delta t}{\boldsymbol{\tau}_{m}}\left(-\left(\tilde{\boldsymbol{v}}^{(n)}-\boldsymbol{v}_{\mathrm{rest}}\right)+\boldsymbol{I}^{(n+1)} \odot \boldsymbol{R}\right)+c \mathcal{N}(0, \Delta t)
$$

370 where $\Delta t$ is the sampling rate (or step size), which was set $\Delta t=1 \mathrm{~ms}$ for this study, $\boldsymbol{\tau}_{m} \in \mathbb{R}^{1 \times N}$ is 371 the membrane time constant, $\boldsymbol{v}_{\text {rest }} \in \mathbb{R}^{1 \times N}$ is the resting potential, $\odot$ refers to Hadamard operation 372 (element-wise multiplication), $\stackrel{-}{-}$ refers to the element-wise division, and $\boldsymbol{R} \in \mathbb{R}^{1 \times N}$ is the input 373 resistance. The term $c \mathcal{N}(0, \Delta t)$ injects spontaneous membrane fluctuations, where $\mathcal{N}(0, \Delta t) \in$ $374 \mathbb{R}^{1 \times N}$ is a Gaussian random vector consisting of $N$ independent Gaussian random variables with 375 mean 0 and variance $\Delta t$, and $c$ is the scaling constant for the amplitude of fluctuations, set as $c=5$ 376 throughout the study.

377 There are two time-varying terms in Eq. 10, the membrane voltage after reset $\left(\tilde{\boldsymbol{v}}^{(n)}\right)$ and input 378 current $\left(\boldsymbol{I}^{(n+1)}\right)$. The voltage reset in the LIF model after action potentials at step $n$ is formulated 379 as

$$
\tilde{\boldsymbol{v}}^{(n+1)}=\boldsymbol{v}^{(n+1)}+\left(\boldsymbol{v}_{\text {reset }}-\boldsymbol{v}^{(n+1)}\right) \odot H\left(\boldsymbol{v}^{(n+1)}-\boldsymbol{\vartheta}\right)
$$

380 where $\boldsymbol{v}_{\text {reset }} \in \mathbb{R}^{1 \times N}$ is the reset potential, $\boldsymbol{\vartheta} \in \mathbb{R}^{1 \times N}$ is the action potential thresholds, and $H(x)$ is 381 the element-wise Heaviside step function. The term $H\left(\boldsymbol{v}^{(n+1)}-\boldsymbol{\vartheta}\right)$ represents the spiking output 
382 activities at step $n+1$. The input current at step $n+1$ is given by

$$
\boldsymbol{I}^{(n+1)}=S^{(n)} \cdot \mathbf{1}+W_{\text {in }} \boldsymbol{u}^{(n+1)}
$$

383 where $\mathbf{1} \in \mathbb{R}^{1 \times N}$ is the column vector with all ones and $S^{(n)}$ is the filtered spike train matrix at 384 step $n$, which follows the iteration

$$
S^{(n)}=S^{(n-1)}+\frac{\Delta t}{T}\left(-S^{(n-1)}+W \odot H\left(\boldsymbol{v}^{(n)}-\vartheta\right)\right)
$$

385 where $T \in \mathbb{R}^{N \times N}$ is the matrix of synaptic decay time constants and $W \in \mathbb{R}^{N \times N}$ is the matrix of 386 synaptic strengths. Here, $W \in \mathbb{R}^{N \times N}$ is a matrix and $H\left(\boldsymbol{v}^{(n)}-\boldsymbol{\vartheta}\right) \in \mathbb{R}^{1 \times N}$ is a row vector. The 387 notation $A \odot \boldsymbol{v}$ refers to element-wise multiplication of matrix $A$ row by row with the row vector $\boldsymbol{v}$.

388 The output at step $n+1$ is computed by

$$
o^{(n+1)}=W_{\text {out }} \boldsymbol{r}^{(n+1)}
$$

389 in which

$$
\boldsymbol{r}^{(n+1)}=\boldsymbol{r}^{(n)}+\frac{\Delta t}{\boldsymbol{\tau}_{r}}\left(-\boldsymbol{r}^{(n)}+H\left(\boldsymbol{v}^{(n+1)}-\boldsymbol{\vartheta}\right)\right)
$$

390 where $\boldsymbol{\tau}_{r} \in \mathbb{R}^{1 \times N}$ is the synaptic decay time constants of firing rate estimate.

391 Training details. In this study, we only used the supervised backpropagation of errors learning 392 algorithm. The loss function $(\mathcal{L})$ is defined in terms of the root mean square error (RMSE) with 393 respect to a task-specific target signal $(\boldsymbol{z})$ and the network output signal $(\boldsymbol{o})$ :

$$
\mathcal{L}:=\sqrt{\left(\sum_{n=1}^{M}\left(z^{(n)}-o^{(n)}\right)^{2}\right)}
$$

394 where $M$ is the total time steps.

395 We used Adaptive Moment Estimation (ADAM) stochastic gradient descent algorithm 42 396 with mini-batch training. The mollifier gradient approximations were employed to address non397 differentiability problem associated with the spiking process (see Mollifier gradient approxi398 mations). The learning rate was set to 0.01 , the batch size was set to 10 , and the first and 
399

400

402 403

404

405

406

408

409

410

411

second moment decay rates were 0.9 and 0.999 , respectively. The trainable parameters include input weights $\left(W_{\text {in }}\right)$, synaptic strengths $(W)$, readout weights $\left(W_{\text {out }}\right)$, synaptic decay time constants $(T)$, membrane time constants $\left(\boldsymbol{\tau}_{m}\right)$, input resistances $(\boldsymbol{R})$, resting potentials $\left(\boldsymbol{v}_{\text {rest }}\right)$, reset voltages $\left(\boldsymbol{v}_{\text {reset }}\right)$, action potential thresholds $(\boldsymbol{\vartheta})$, and synaptic decay time constants for firing rate estimates $\left(\boldsymbol{\tau}_{r}\right)$.

A nonlinear projected gradient method was used to constrain parameters within the biologically realistic ranges described in Supplementary Table 1. A linear projection map forces some solutions to be projected on the boundary. That is, there are always some units whose parameters take the min and max values of the constraint. On the other hand, a nonlinear projection guarantees that no values are on the boundary almost surely, a more realistic situation to consider. Specifically, to bound a parameter $p$ at iteration $i+1$ into the range $\left[p_{\min }, p_{\max }\right]$, we have

$$
\tilde{p}_{i+1}=\sigma\left(p_{i+1}\right) \cdot\left(p_{\max }-p_{\min }\right)+p_{\min }
$$

where $\tilde{p}_{i+1}$ is the projected solution of parameter $p$ at iteration $i+1, p_{i+1}$ is the unconstrained solution given by the gradient descent algorithm at iteration $i+1, p_{\max }$ and $p_{\min }$ are the maximum and minimum values of parameter $p$, and $\sigma(x)$ is the sigmoid function, defined as

$$
\sigma(x):=\frac{1}{1+\exp (-x)}
$$

We initialized all parameters, except the input weights $\left(W_{\text {in }}\right)$, as samples from the standard Gaussian distribution with zero mean and unit variance, whereas the input weights were drawn from Gaussian distribution with zero mean and variance 400. This is because our input signals were bounded within the range $[-1,1]$, insufficient to bring the membrane voltage from the resting potential above the action potential threshold. Hence, to accelerate training, it was necessary to make sure units were excited by the input signals in the first place. The synaptic strength matrix $(W)$ was also initialized sparse, with the percentage of connectivity being only $20 \%$. We say the network successfully did the task if the output signal hits above +0.8 (or below -0.8 ) if the target output is +1 (or -1 ). We stopped training when the loss $(\mathcal{L})$ is less than 15 and the accuracy over 100 trials is above $95 \%$. 
The method proposed by Song et al. [29] was used to impose Dale's principle with separate 424 excitatory and inhibitory populations. The synaptic connectivity matrix $(W)$ in the model was 425 parametrized by

$$
\tilde{W}_{i+1}=\left[W_{i+1}\right]_{+} \cdot D
$$

426 where $\tilde{W}_{i+1}$ is the resulted matrix that encoded separate populations at update step $i+1, W_{i+1}$ 427 is the solution given by the gradient descent algorithm at step $i+1$, and $[\cdot]_{+}$is the rectified 428 linear unit (ReLU) operation applied at the end of each update step. The ReLU operation is to 429 ensure that entries of the matrix are always non-negative before multiplied by the matrix $D$, as the 430 negative weight connections update from gradient descent are pruned by the end of each update. 431 The diagonal matrix $\left(D \in \mathbb{R}^{N \times N}\right)$ encode +1 for excitatory units and -1 for inhibitory units.

432 The value of matrix $(D)$ was randomly assigned before training according to a preset proportion 433 between inhibitory and excitatory units, and the value $D$ was fixed through the whole training 434 process. The I/E units proportion in this study was $20 \%$ to $80 \%$.

435 In order to capture the biologically realistic dynamics of SNNs, the temporal resolution $(\Delta t)$ 436 was set to be no longer than the duration of absolute refractory period to ensure that the spiking 437 activities are not affected by the numerical integration process. Therefore, we set $\Delta t=1 \mathrm{~ms}$ during 438 training. Due to the vanishing gradient problem occurring in training RNNs [43], with $\Delta=1 \mathrm{~ms}$, 439 it is impossible to train tasks with duration longer than 1 second (i.e., $M>1000$ ). It is notable 440 that in the above formulation, only membrane time constant $\left(\tau_{m}\right)$ and synaptic time decay $(\tau)$ are 441 dependent on the sampling rate ( $\Delta t$; Eq. 10 and Eq. 13). Hence, after the models are trained, 442 we can make sampling rate $(\Delta t)$ smaller (i.e., having finer temporal resolution) while still keeping 443 the same dynamics of the trained networks. Increasing $\Delta t$ by a factor is equivalent to decreasing $\tau$ 444 and $\tau_{m}$ altogether by the same factor, as $\tau$ and $\tau_{m}$ are inversely proportional to $\Delta t$ in Eq. 10 and 445 Eq. 13. Hence, to train a network performing tasks with duration longer than 1 second, we need 446 to make the temporal resolution coarser (i.e., increasing $\Delta t$ by a factor $s$ ) so that with the same 447 trainable range of time steps (i.e., a fixed $M \leq 1000$ ), the duration of task becomes longer by the 448 same factor $s$. This "decrease in temporal resolution" can be interpreted as shortening $\tau$ and $\tau_{m}$ 449 instead of an actual decrease in temporal resolution. Applying this trick enables us to train tasks 450 with arbitrary duration by re-scaling the ranges of $\tau$ and $\tau_{m}$ into a smaller one while still making 
451 the spiking activities biologically realistic. In practice, we simply scaled down $\tau$ and $\tau_{m}$ by a factor $452 s=3$ with a fixed number of time steps $(M)$, and later during the testing stage, we re-scaled $M, \tau$ 453 and $\tau_{m}$ up by the same factor $s$.

454 Mollifier gradient approximations. In the above formulation, the Heaviside step function $H(x)$ 455 is not continuous. As a result, the loss function $\mathcal{L}$ is not differentiable. This poses the major problem 456 when applying the traditional backpropagation algorithm for training neural networks, because the 457 backpropagation algorithm uses gradient descent methods that require the function being minimized 458 to be differentiable, or at least to be continuous. However, the derivative of Heaviside step function $459 H(x)$ is Dirac Delta function $\delta(x)$, which is 0 everywhere except at 0 , where the function value is $460 \infty$. It is difficult to use this derivative for the gradient descent methods because the value of the 461 gradients is 0 almost everywhere. To address the discontinuity problem, we employed mollifier gradient method proposed by

463 Ermoliev et al. [27]. The method can be applied to any strongly lower semicontinuous functions 464 to find local minima following an iterative gradient descent in which the gradients change over 465 iterations based on averaged functions derived from the original objective function. The family of 466 averaged functions $f_{\varepsilon}$ of function $f$ is defined by convolution of $f$ with a mollifier: $\psi_{\varepsilon}$

$$
f_{\varepsilon}(x):=\int_{\mathbb{R}^{n}} f(x-z) \psi_{\varepsilon}(z) d z=\int_{\mathbb{R}^{n}} f(x) \psi_{\varepsilon}(x-z) d z=f * \psi_{\varepsilon}(x)
$$

467 468

where $\psi_{\varepsilon} \in\left\{\psi_{\varepsilon}: \mathbb{R}^{n} \rightarrow \mathbb{R}_{+}, \varepsilon>0\right\}$, a family of compactly supported (generalized) functions named mollifiers that satisfy

$$
\int_{\mathbb{R}^{n}} \psi_{\varepsilon}(x) d x=1, \quad \lim _{\varepsilon \rightarrow 0} \psi_{\varepsilon}(x)=\lim _{\varepsilon \rightarrow 0} \varepsilon^{-n} \psi_{\varepsilon}(x / \varepsilon)=\delta(x)
$$

It was shown that for any strongly lower semicontinuous functions $f$, the averaged functions $f_{\varepsilon}$ epi-converge to $f$ as $\varepsilon \rightarrow 0$, a type of convergence that preserves the local minima and minimizers.

71 Therefore, it is possible to use the gradients of averaged functions to minimize the original lower semicontinuous functions and find the local minima. We used the conventional family of mollifiers 
473 obtained by normalizing a probability density function $\psi$ :

$$
\psi_{\varepsilon}(z):=\frac{\psi(z / \varepsilon)}{\varepsilon^{n}}
$$

474 In our case, $n=1$ as the domain of $H(x)$ is the real line:

$$
H_{\varepsilon}(x):=\frac{1}{\varepsilon} \int_{-\infty}^{\infty} H(x-z) \psi(z / \varepsilon) d z
$$

475 For any $\varepsilon>0$, the gradient of $H_{\varepsilon}(g(x))$ with respect to parameter $p$ is given by

$$
\nabla_{p} H_{\varepsilon}(g(x))=\frac{1}{\varepsilon} \psi(g(x) / \varepsilon) \nabla_{p} g(x)=\psi_{\varepsilon}(g(x)) \nabla_{p} g(x)
$$

476 where $\psi$ is some symmetric density function and $g(x)$ is any function with $\mathbb{R}$ as its codomain. Since 477 our goal was not to find a local minimum $x^{*}$ that satisfies the optimality condition $\lim _{\varepsilon \rightarrow 0}\left\|\nabla f_{\varepsilon}\left(x^{*}\right)\right\|=0$ 478 as defined by Ermoliev et al., but rather to minimize the loss function for its value to be sufficiently 479 small so that the network can perform the task correctly, we did not vary the gradients during the 480 minimization process. Instead, we fixed an approximation of the gradient and used the approxi481 mation throughout the training process. We chose the normalized box function, i.e., the density 482 function of uniform distribution $\mathcal{U}(-\varepsilon / 2, \varepsilon / 2)$, as the kernel,

$$
\psi(x):= \begin{cases}\frac{1}{\varepsilon} & \text { for } x \in[-\varepsilon / 2, \varepsilon / 2] \\ 0 & \text { otherwise }\end{cases}
$$

483 and fixed $\varepsilon=5$.

484 We found no difference in the trained models with different choices of $\varepsilon$, as long as the value 485 was large enough to keep the gradients active so that gradients did not vanish through time steps. 486 There was also no difference between models trained with fixed $\varepsilon$ and those trained with the original 487 scheme in Ermoliev et al. where $\varepsilon \rightarrow 0$ as the number of iterations increases. The purpose for fixing 488 the value of $\varepsilon$ was to compare the training epochs (iterations) among the retraining paradigms (see 489 Fig. 3) with the same gradient. 
490 Re-training models for DMS task. To test whether intrinsic properties optimized for one WM

491 task are generalizable to other tasks that also require WM, we re-trained our models to perform

492 the DMS task with all intrinsic properties fixed. In contrast to the training paradigm described

493 in the previous sections, the trainable parameters for re-training only include input weights $\left(W_{\text {in }}\right)$,

494 synaptic strengths $(W)$, and readout weights $\left(W_{\text {out }}\right)$. Each of the 20 RNNs trained for each of the

495 four tasks (non-WM integration, delayed integration, DMS, and DIS tasks) used in this study was

496 re-trained to perform the DMS task.

497 To test whether synaptic decay time constants $(\tau)$ and membrane time constants $\left(\tau_{m}\right)$ are the

498 most crucial parameters for transferability of WM tasks, we repeated the re-training procedure

499 with both $\tau_{m}$ and $\tau$ either fixed or optimized for the non-WM RNNs. The RNNs optimized to

500 perform the context-based input integration task were used for re-training under two schemes: the

501 tuned scheme and the frozen scheme. For the tuned scheme, the trainable parameters include input

502 weights $\left(W_{\text {in }}\right)$, synaptic strengths $(W)$, readout weights $\left(W_{\text {out }}\right)$, synaptic decay time constants $(T)$,

503 membrane time constants $\left(\boldsymbol{\tau}_{m}\right)$, and synaptic decay time constants for firing rate estimates $\left(\boldsymbol{\tau}_{r}\right)$.

504 For the frozen scheme, the trainable parameters include input weights $\left(W_{\text {in }}\right)$, synaptic strengths

$505(W)$, readout weights $\left(W_{\text {out }}\right)$, input resistances $(\boldsymbol{R})$, resting potentials $\left(\boldsymbol{v}_{\text {rest }}\right)$, reset voltages $\left(\boldsymbol{v}_{\text {reset }}\right)$,

506 and action potential thresholds $(\boldsymbol{\vartheta})$.

507 Units function analysis. For Fig. 4, we manipulated $\tau_{m}$ and $\tau$ during different epochs of the

508 DMS task paradigm to investigate if fast membrane and slow synaptic dynamics are responsible for

509 WM maintenance. For each of the RNNs trained from the DMS task, we first divided the population

510 into two subgroups based on their $\tau_{m}$ values. The short $\tau_{m}$ group contained units whose $\tau_{m}$ was

511 smaller than the median value of $\tau_{m}$ of all units in the RNN, while the long $\tau_{m}$ group contained

512 units whose $\tau_{m}$ was greater than the median value. The average median value of $\tau_{m}$ across all 20

513 models was $19.64 \pm 2.45 \mathrm{~ms}$. During each of the four epochs (fixation, first stimulus, delay, and

514 second stimulus), we inhibited the two $\tau_{m}$ subgroups separately by hyperpolarizing them and then

515 assessed the task performance. The hyperpolarization was done by setting the membrane voltage

$516 v=-100 \mathrm{mV}$ for the intended subgroup of units. Similar to the training stage, we say that the

517 network successfully did the task if the output signal hits above +0.8 (or below -0.8 ) if the target

518 output is +1 (or -1 ). If the target output is between -0.8 and +0.8 , the network is considered 
519 having no response. If the output signal is above +0.8 (or below -0.8 ) while the target output is $520-1($ or +1$)$, we say that the network gives an incorrect response.

521 We conducted a similar analysis based on two subgroups of synapses derived from a quartile

522 split of synaptic decay time constant $(\tau)$. The short $\tau$ group contained synapses whose $\tau$ was

523 smaller than the 25th percentile of all $\tau$ in the RNN, while the long $\tau$ group contained synapses

524 whose $\tau$ was greater than the 75 th percentile. The average 25 th percentile across all 20 models was

$52525.36 \pm 2.40 \mathrm{~ms}$, and the average $75 \mathrm{th}$ percentile was $66.18 \pm 1.17 \mathrm{~ms}$. The targeted subgroup of

526 synapses was suppressed by setting the connection strength $w=0$ during each of the four epochs 527 of DMS task.

\section{Code availability}

529 The implementation of our framework and the codes to generate all the figures in this work are

530 available at https://github.com/y-inghao-li/SRNN/

\section{Data availability}

532 The trained models used in the present study are available as MATLAB-formatted data at https:

533 //github.com/y-inghao-li/SRNN/ 


\section{Supplementary Table}

\begin{tabular}{cccc}
\hline Parameter name & Symbol & Minimum & Maximum \\
\hline \hline Input resistance & $R$ & $5 \mathrm{M} \Omega$ & $1000 \mathrm{M} \Omega$ \\
\hline $\begin{array}{c}\text { Membrane time } \\
\text { constant }\end{array}$ & $\tau_{m}$ & $5 \mathrm{~ms}$ & $50 \mathrm{~ms}$ \\
\hline $\begin{array}{c}\text { Action potential } \\
\text { threshold }\end{array}$ & $\vartheta$ & $-50 \mathrm{mV}$ & $-30 \mathrm{mV}$ \\
\hline Resting potential & $v_{\text {rest }}$ & $-80 \mathrm{mV}$ & $-60 \mathrm{mV}$ \\
\hline $\begin{array}{c}\text { Reset voltage } \\
\text { value }\end{array}$ & $v_{\text {reset }}$ & $v_{\text {rest }}-10 \mathrm{mV}$ & $v_{\text {rest }}-1 \mathrm{mV}$ \\
\hline Synaptic decay time & $\tau$ & $5 \mathrm{~ms}$ & $100 \mathrm{~ms}$ \\
\hline
\end{tabular}

Supplementary Table 1: Parameter values used for this study. To keep the constraint $v_{\text {rest }}>$ $v_{\text {reset }}$, we trained the afterhyperpolarization (AHP) potential with range from $-10 \mathrm{mV}$ to $-1 \mathrm{mV}$, so the value of $v_{\text {reset }}$ is dependent upon the value of $v_{\text {rest }}$. 